\title{
Computational Models of the Heart and Their Use in Assessing the Actions of Drugs
}

\author{
Denis Noble $e^{1, *}$ \\ ${ }^{1}$ Department of Physiology, Anatomy and Genetics, University of Oxford, Parks Road, Oxford, OX1 3PT, UK
}

Received July 20, 2007

\begin{abstract}
Models of cardiac cells are sufficiently well developed to answer questions concerning the actions of drugs on repolarization and the initiation of arrhythmias. These models can be used to characterize drug-receptor action profiles that would be expected to avoid arrhythmia and so help to identify drugs that may be safer. Several examples of such action profiles are presented here, including a recently-developed blocker of persistent sodium current, ranolazine. The models have also been incorporated into tissue and organ models that enable arrhythmia to be modelled also at these levels. Work at these levels can reproduce both re-entrant arrhythmia and fibrillation.
\end{abstract}

Keywords: cardiac cell model, cardiac organ model, repolarization, early after-depolarization (EAD), delayed after-depolarization (DAD)

\section{Introduction}

Computational models of heart cells have a long history of development during which they have become perhaps the most detailed cell models available today. Over 100 such models are listed on the CellML website (www.cellml.org), which means that they can be downloaded and used immediately with software that is CellML compatible (e.g. http://cor.physiol.ox.ac.uk/).

The first models using equations for the kinetics of ion channels were created in $1960(1,2)$, and they were based on early experimental work on the potassium channels in heart muscle $(3-5)$, and on the groundbreaking work of Hodgkin and Huxley who created the first nerve axon model (6).

The experiments with Otto Hutter led to the first classification of potassium channels in the heart into those showing very rapid inward-rectification $\left(i_{\mathrm{K} 1}\right)$ and those showing delayed (time-dependent) outward rectification $\left(\mathrm{i}_{\mathrm{K}}\right)$. As shown in Fig. 1, these two types of channel play very different roles in depolarization and repolarization. Because $\mathrm{i}_{\mathrm{K} 1}$ changes rapidly, the

*Corresponding author. denis.noble@physiol.ox.ac.uk Published online in J-STAGE

doi: $10.1254 /$ jphs.CR0070042

Invited article trajectory first follows its voltage-dependence. As a consequence, the potassium conductance becomes very low at the beginning of a ventricular action potential. This can be seen as an energy-conserving device since very small sodium and calcium conductances are then sufficient to maintain the long plateau of the action potential, and less energy is required to restore the gradients of all these ions following each heartbeat.

Repolarization depends on the slow activation of $i_{K}$ channels. As I will show later in this article, the repolarization process is fragile. Many drugs can interfere with repolarization. In fact such side-effects of drugs on the heart are frequent and serious. According to some estimates around $40 \%$ of all new pharmaceutical compounds have such effects. They can cause serious arrhythmias that may be fatal. The cost to the industry is large. Withdrawal of a drug after approval represents a lost investment of more than $\$ 1$ billion before one even begins to count lost sales and share values. A solution to this problem would therefore be beneficial to everyone. It would make the industry more successful, since many of the compounds withdrawn, or which never even make it to the market for this kind of reason, are effective drugs in other respects. Moreover, the side effects may be experienced by much less than $1 \%$ of the patient population. For the vast majority of patients the drugs would be beneficial. Rescuing such drugs might therefore be worthwhile and discovering combinations of 

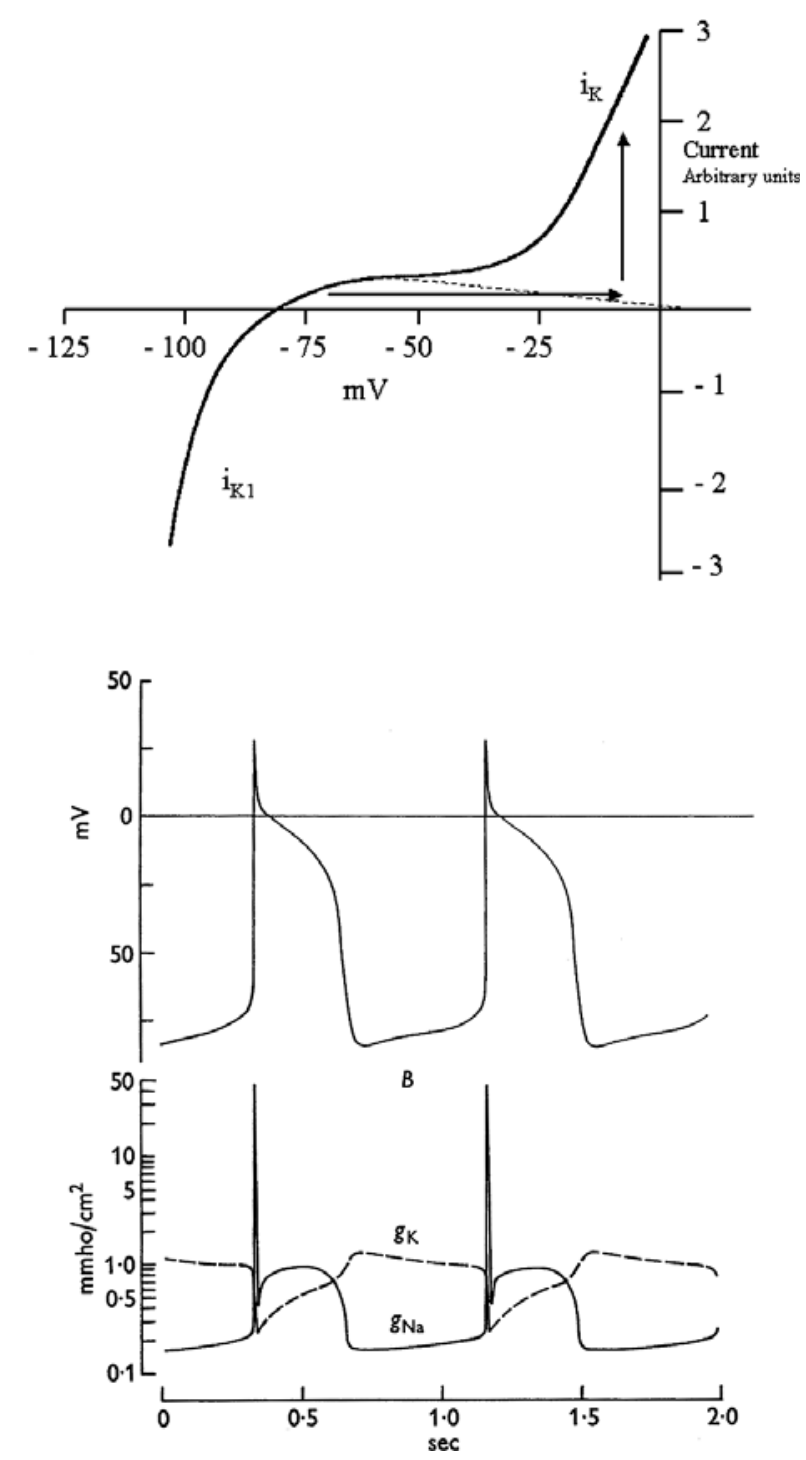

Fig. 1. Top: The first analysis of potassium channel currents in the heart and their incorporation into a heart cell model. Redrawn from Ref. 4. The solid line shows the total membrane current recorded in a Purkinje fiber in a sodium-depleted solution. The inward-rectifying current was identified as $i_{\mathrm{K} 1}$, which is extrapolated here as nearly zero at positive potentials. The outward-rectifying current, $i_{K}$, is now known to be mostly formed by the component $i_{\mathrm{Kr}}$. The horizontal arrow indicates the trajectory at the beginning of the action potential, while the vertical arrow indicates the time-dependent activation of $i_{K}$ that initiates repolarization. Bottom: Sodium and potassium conductance changes computed from the first biophysically-detailed model of cardiac cells (2). Two cycles of activity are shown. The conductances are plotted on a logarithmic scale to accommodate the large changes in sodium conductance. Note the persistent level of sodium conductance during the plateau of the action potential, which is about $2 \%$ of the peak conductance. Note also the rapid fall in potassium conductance at the beginning of the action potential. This is attributable to the properties of the inward rectifier $i_{\mathrm{K} 1}$, and it helps to maintain the long-duration of the action potential and helps to promote energy-conservation by greatly reducing the ionic exchanges involved. The current $i_{K}$ is then responsible for repolarization. The arrows correspond to those shown in the top panel. These insights into the main potassium current changes have been incorporated into all subsequent models of cardiac cells. actions that avoid the problem could improve the selection of lead compounds at an early stage.

It would benefit health care for other reasons too. One reason that drug prices are high is that health care systems have to bear the cost of industry failures as well as industry successes. Unfortunately, the failures greatly outnumber the successes, by around 50:1 according to some estimates. One analyst in the industry told me: "All you have to do is to decrease our attrition rate from around $98 \%$ to $96 \%$ and you would make us twice as successful." So, simulation does not have to be $100 \%$ successful to have a major impact on drug development. Even if just a fraction of the insights gained are valuable, that could be enough to make a significant difference.

It is also important to emphasize that these insights can be of many different kinds. The dream of being able to use computer models as though they were the equivalent of experimental animals, or even substitute virtual humans, is a long way off. Even the most sophisticated of computer models of the heart may represent the functionality of only $2 \%$ of the gene and protein mechanisms involved, and many of those mechanisms need further refinement. Therefore, we are not yet in the position of the aircraft industry, which can simulate a whole aircraft with reliable predictive capability on its ability to fly and to carry out all the other functions required. However, that analogy also provides the clue to a more positive message. Insights from computer models of aircraft were valuable even before they replaced wind-tunnel experiments. Improving our quantitative understanding of complex interactions in biological systems, and refining the experimental approaches necessary to further refine the models, can have valuable practical spin-offs. In this article, I will show the extent to which simulation can help to address the cardiac arrhythmia problem.

\section{Current methods for screening against arrhythmia}

Of course, people have looked for biological markers to give at least early warning of possible cardiac problems. However, at present we are using very unreliable markers: QT and hERG. QT is the interval between the rapid QRS complex of the electrocardiogram, corresponding to the sharp depolarization wave as excitation spreads through the ventricle, and the $\mathrm{T}$ wave, which corresponds to repolarization. To a first approximation, therefore, the QT interval is a measure of the duration of the ventricular action potentials. Since some of the drugs that cause arrhythmic side-effects prolong the action potential, measuring QT, perhaps together with other markers, such as action on the repolarizing current $i_{\mathrm{Kr}}$ or one of its proteins hERG, 
might identify the problem compounds. Measuring QT, right down to the last millisecond or two, has therefore become a refined technical art with many variations on how exactly to measure it. The $\mathrm{T}$ wave is hardly ever kind enough to give you a completely obvious point of measurement, so how to correct those measurements for unrelated variations, and how to automate all of this, has become necessary.

Unfortunately, the link between prolonged QT and arrhythmia is neither necessary nor sufficient. Some drugs cause arrhythmia without prolonging QT and some drugs that prolong QT do not cause arrhythmia. The QT interval is therefore a seriously flawed marker. A similar problem applies to hERG. Some drugs that target hERG $\left(\mathrm{i}_{\mathrm{Kr}}\right)$ do not cause arrhythmia. That depends on what other actions they have. Some compounds that block $i_{\mathrm{Kr}}$ actually shorten the action potential because of these additional effects.

Can we do better? That is the question I am going to try to answer in this article.

\section{Origin of the problem}

It is always best to understand a biological problem before trying to find ways to avoid it. The reason that action potential duration is important is that repolarization is a fragile process. It can easily fail, and when it does so, the action potential is followed by one or many oscillations. These are called early-after depolarizations (EADs). We will also encounter late afterdepolarizations (DADs) later in this article.

Why is repolarization fragile, while depolarization is so robust? The answer lies in the quantities of electrical current involved. Depolarization is generated by a very large (approximately 100 fold) increase in membrane conductance as the dense population of sodium channels is activated. Once the threshold for initiating the process has been reached, it is exceedingly difficult to stop it from running its full course. Depolarization is therefore extremely robust. By contrast, repolarization is brought about by very tiny currents resulting from a fine balance between almost equal depolarizing and repolarizing factors. Not only are the currents involved small, they are even smaller than one might expect because, once the fast depolarization phase is complete, the net membrane conductance actually falls well below its resting value, a fact that was first discovered by Weidmann (7). This was a surprise since it is exactly the opposite result from that obtained during nerve excitation (8). It was the reconstruction of Cole and Curtis' experimental result that formed one of the great successes of the Hodgkin-Huxley model of the nerve impulse (6). Reconstruction of Weidmann's experi- mental data has also been a criterion for validating cardiac cell models $(2,9,10)$.

The reason for this difference between heart and nerve is an evolutionary compromise of the form that I have called 'nature's pacts with the devil' (11). These are what we, with hindsight, would call design faults, but which, from an evolutionary point of view, are the inevitable price paid for many successful developments. They resemble Faust's pact with the devil in which Faust secured years of unlimited knowledge and power, but at the price of giving the devil his soul. The key to this kind of pact is that it is eventually fatal but for a long time it is of great benefit. This is just the kind of pact that nature stumbles upon when it finds a good combination of genes to transmit a function, at a price that may eventually be fatal. Evolution may take little notice of the fatality in individuals, particularly if it occurs well after the reproductive period of life. And evolution certainly did not anticipate the coming of the pharmaceutical industry.

The long-lasting cardiac action potential is a consequence of such an evolutionary compromise in the development of potassium currents in the heart. As we have seen, these channels can be divided into two classes: channels that close on depolarization, $i_{\mathrm{K} 1}$, and channels that open during depolarization, including the various components of $i_{K}$, and of the transient outward current, $\mathrm{i}_{\mathrm{to}}$. At rest, $\mathrm{i}_{\mathrm{K} 1}$ is switched on and holds the resting potential at a very negative level, where the other $\mathrm{K}^{+}$currents are switched off. On depolarization $\mathrm{i}_{\mathrm{K} 1}$ rapidly switches off, while the other currents take time to activate and to cause repolarization.

The biological advantage of this potassium channel system is that it saves energy (see Introduction). The energy required to pump ions back again during each cardiac cycle is minimized. Even with this economy, around $20 \%$ of the energy consumption of the heart is attributable to ion pumping. That figure would have been much larger without the $i_{\mathrm{K} 1}$ mechanism. That is the good side of nature's Faustian pact.

The bad side is that hERG, one of the proteins that forms the main component of $i_{K}$, is highly promiscuous. The channel can be blocked by many pharmaceutical compounds. When that happens, repolarization fails and the action potential is followed by one or more waves of depolarization (Fig. 2). These can trigger cardiac arrhythmia that in some cases is fatal.

Figure 2 was produced by running the Noble_model 2000 CellML file within the modelling software COR (http://COR.physiol.ox.ac.uk). In this model 65\% block of $i_{\mathrm{Kr}}$ is sufficient to prevent smooth repolarization and to initiate a series of EADs. One of the inward currents involved in each depolarization is the L-type calcium 


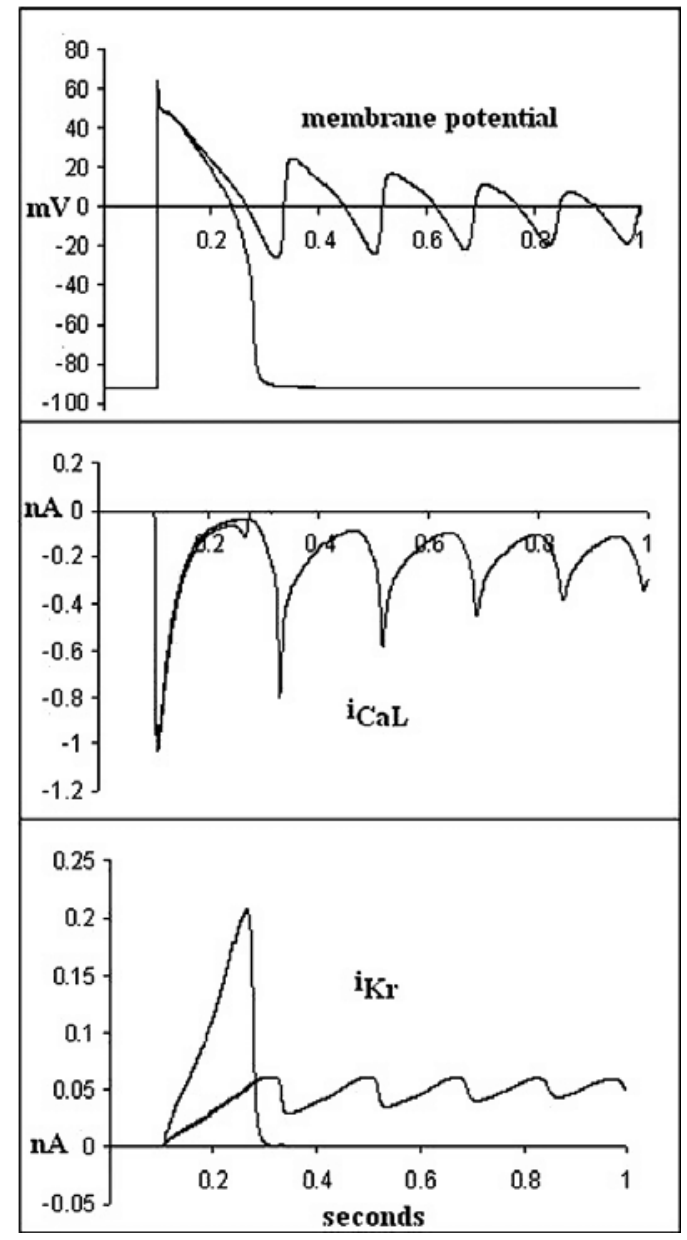

Fig. 2. Top: action potentials computed using a model of a guineapig ventricular cell. Middle: calcium current $i_{\mathrm{CaL}}$. Bottom: potassium current $i_{\mathrm{Kr}}$. A $65 \%$ block of the fast component of $i_{K}$ prevents repolariztion and generates multiple after-depolarizations (12).

current, $\mathrm{i}_{\mathrm{CaL}}$. The middle trace shows multiple reactivations of this current during each oscillation of membrane potential.

Many factors can interact with drugs to make this problem worse. These include genetic factors (see Fig. 3), such as mutations in sodium, potassium, and calcium channel genes, that predispose people to repolarization failure $(13-15)$. This is the main explanation for the fact that drugs have this side effect do so in only a small fraction of the population. In principle, it should become possible to screen for such genetic predispositions to exclude such patients in clinical trials and to avoid treating them with drugs that interact in this way.

\section{Avoiding the problem}

Can drugs be designed to avoid this kind of problem?
Clearly, there is no way in which we can correct nature's 'mistake' in evolving repolarization channels that are blocked by so many drugs. Dreams of doing so by genetic manipulation are not just unimaginably improbable dreams, they would also be unethical. We can never be sure that a gene that we have identified with one particular function might not also be involved in many others we do not know about (11). So, it is quite possible that the molecular properties that enable $i_{\mathrm{Kr}}$ to perform its role in the heart are also those that predispose it to drug sensitivity. Therefore, the way forward is to design better drugs. That this can be done is illustrated in Fig. 4 . This shows the same computation as in Fig. 2, but with the addition of a computation in which $65 \%$ block of the potassium channel was combined with $20 \%$ block of the $\mathrm{L}$ type calcium channels. The result is a smooth repolarization with no signs of EADs. Clearly, a multireceptor drug with this combination of properties would be expected to avoid arrhythmia. Such compounds exist. This particular computation was of a compound BRL32872 that has exactly this profile of action (17). Amiodarone, which is a multi-site drug, also includes this profile, to which we can also add inhibition of sodium-calcium exchange (18). This suggests that there may be many combinations of drug actions that could be effective. Drugs that include actions on persistent sodium current $(19-22)$ are also in this category $(23-$ 25) - see Fig. 5.

\section{Multiple cellular mechanisms of arrhythmia}

EADs form just one of the several known cellular mechanisms of arrhythmia. DADs are a second mechanism. These consist in spontaneous depolarizations arising after repolarization is complete, and they are known to be caused by intracellular calcium oscillations in conditions of intracellular sodium overload. Such conditions are found in a variety of pathological states including heart failure and ischemic heart disease. The initial causes vary but the common mechanism is reduced energy available to pump sodium out of the cell via $\mathrm{Na}^{+}-\mathrm{K}^{+}$ATPase (the sodium pump). Intracellular sodium therefore rises above its normal range (around $5-10)$ into a range $(12-20 \mathrm{mM})$ that can cause arrhythmias. The processes involved are now understood well enough to model them (27). The rise in intracellular sodium acts via sodium-calcium exchange to cause a rise in intracellular calcium. Above a certain threshold, this can stimulate release of calcium from the sarcoplasmic reticulum via the same mechanism that underlies normal EC coupling, that is, calcium-induced calcium release. Finally, the oscillatory changes in intracellular calcium induce oscillatory inward current 

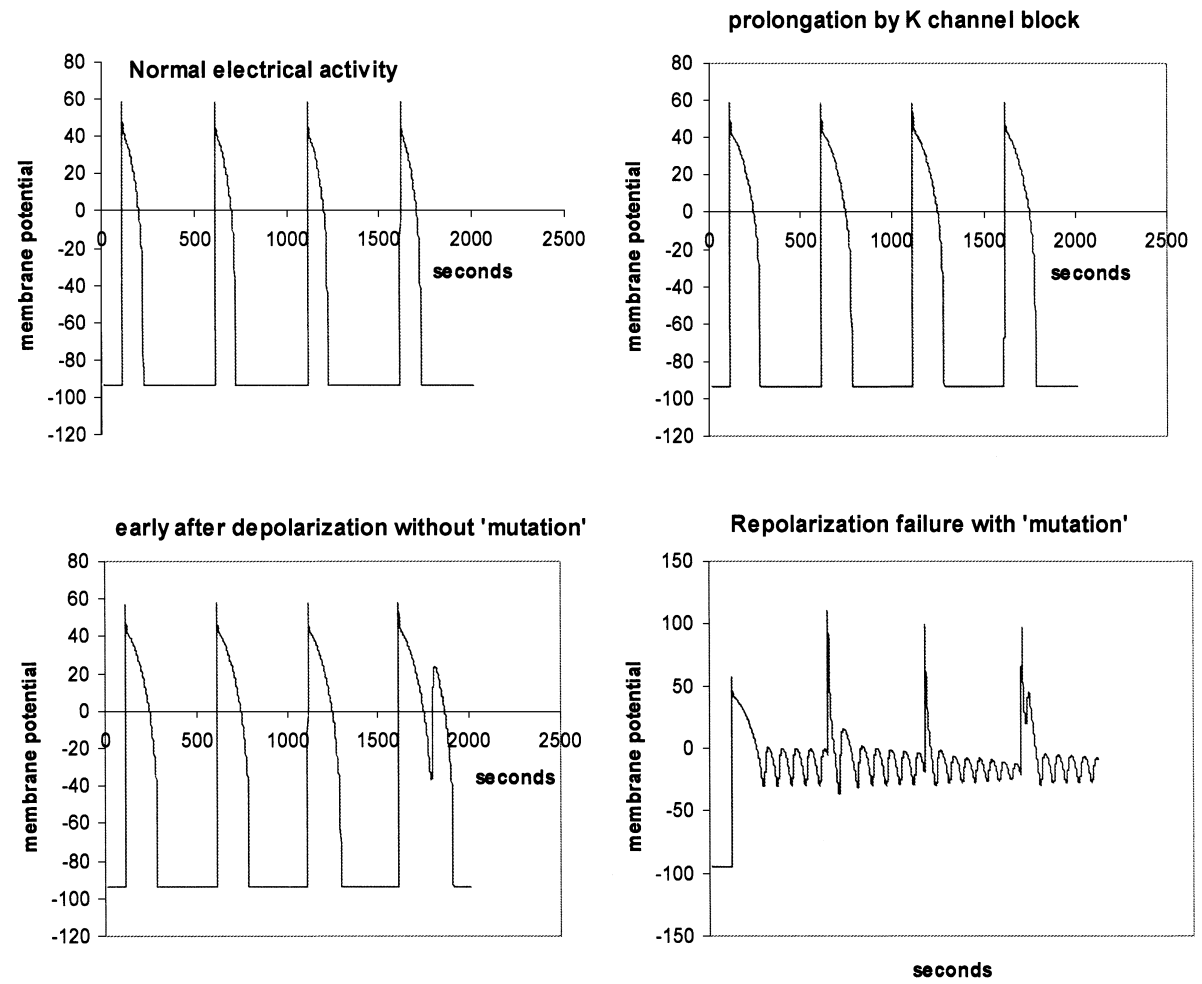

Fig. 3. Example of the use of simulation to understand the non-linear interaction between the effects of mutations affecting repolarization and drug actions. A guinea-pig cardiac ventricular cell model (16) was used to compute the threshold for initiation of repolarization failure for a known effect of one of the mutations predisposing towards arrhythmia. A voltage shift of $23 \mathrm{mV}$ in the inactivation curve for the sodium channel (SCN5) was sufficient to initiate occasional repolarization failure in the form of single after-depolarizations (seen in the bottom left panel). The computations were then repeated (right) after simulating the action of a class III drug ( $\mathrm{i}_{\mathrm{Kr}}$ block by $90 \%$, top right). The threshold 'genetic mutation' shift was then reduced to $17 \mathrm{mV}$ and it resulted in much more severe multiple EADs (bottom right). This kind of computation explains several otherwise puzzling phenomena: 1. The interaction of both drug and mutation effects with the rest of the cellular components is highly non-linear. 2 . Drug and mutation effects can interact in a way that would explain why a small fraction of the population may be particularly susceptible to drug-induced arrhythmia, as found in many clinical trials. 3. Below the threshold mutation effect, there is almost no effect on repolarization time, which would explain why, in the great majority of patients, no QT prolongation is seen.

via sodium-calcium exchange that, if large enough, can trigger extra action potentials (ectopic beats). Figure 6 shows an example of this phenomenon in an atrial cell model.

Only some of the cell models succeed in reproducing this phenomenon, which depends critically on the equations used to represent calcium-induced calcium release. There are still many gaps in our knowledge of this process (29), particularly concerning the role of sub-sarcolemmal spaces in which the free calcium concentration may reach levels much higher than in the bulk cytosol. This is an area where modelling needs to make much more progress by refining our understanding of the EC coupling process, the role of 'fuzzy' spaces, and the mechanisms of calcium signalling within the cell (30).

This is a long cascade of events and therapeutic intervention can therefore be targeted at several different points. Some pharmaceutical approaches focus on the final stage, the generation of depolarizing electric current by sodium-calcium exchange. Inhibitors of sodium-calcium exchange have been developed. The object in this case is either to inhibit the electric current generated by the exchanger or to reduce its contribution to calcium overload in conditions in which it operates in reverse mode. Neither approach has yet proven effective.

It might therefore be better to intervene at an earlier stage in the cascade and attempt to limit one of the earlier stages, that is, sodium overload. This is the approach used in a new class of cardiac drugs that inhibit the persistent sodium current while having little or no effect on the peak sodium current. The first example of such a drug is ranolazine (25). As we have already seen in Fig. 5, inhibition of persistent sodium current is the basis of this compound's ability to avoid inducing EADs. Figure 7 shows that it would also be expected to reduce sodium loading. 


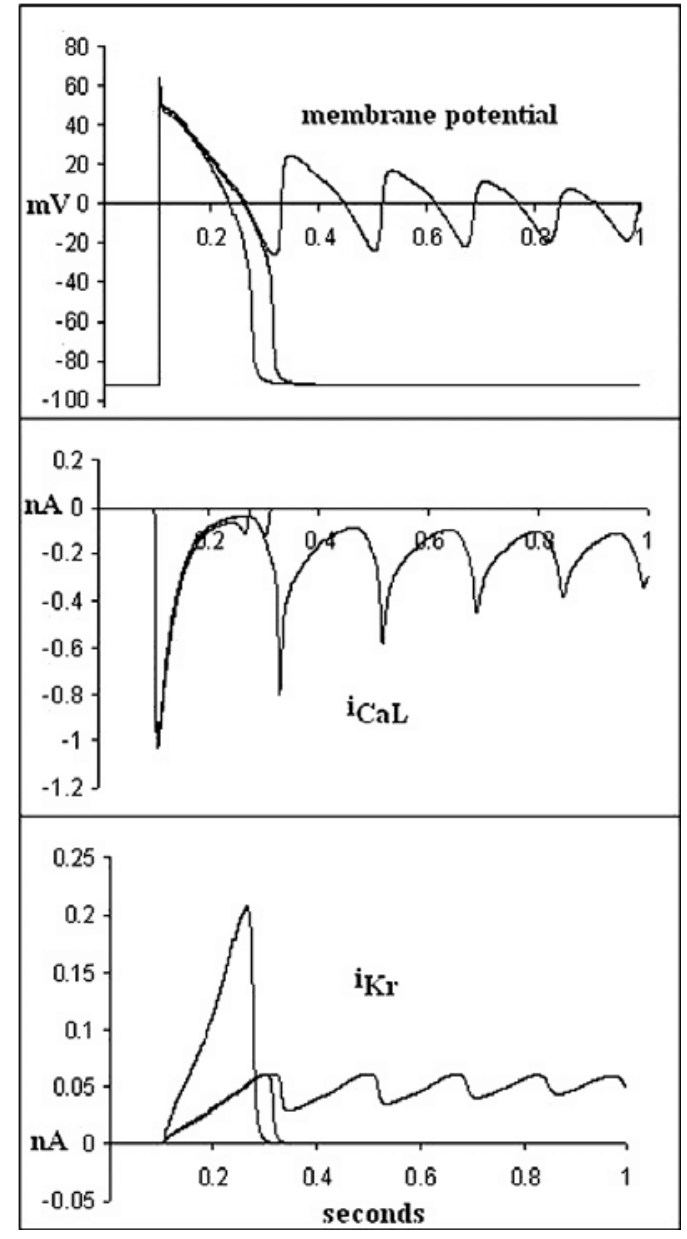

Fig. 4. The computation shown in Fig. 2 has been extended by including $20 \%$ inhibition of the $\mathrm{L}$ type calcium current as well as $65 \%$ inhibition of $\mathrm{i}_{\mathrm{Kr}}$. The result is almost the same degree of action potential prolongation but with smooth repolarization. This reproduces the action of BRL-32872 (16).

The top traces are experimental recordings from the work of Boyett et al. (31) showing the rise in intracellular sodium in a sheep Purkinje fibre on stimulating repetitively after a long period of rest. Over a period of $400 \mathrm{~s}$ intracellular sodium activity increases from under $6 \mathrm{mM}$ to about $8 \mathrm{mM}$. The lower traces show much the same degree of rise in internal sodium in the ventricular cell model. A repeat of the computation with the persistent sodium current fully blocked reduces the sodium loading, in this case by about $30 \%$.

This mechanism is thought to be the basis of the therapeutic effect of this compound in cardiac ischemia, since one of the main causes of arrhythmia in this condition is attributable to sodium overload.

Figure 8 shows that a relatively small increase $(25 \%)$ in the persistent sodium current can result in instability due to sodium overload. In this simulation, a failure of repolarization leads to a large rise in intracellular

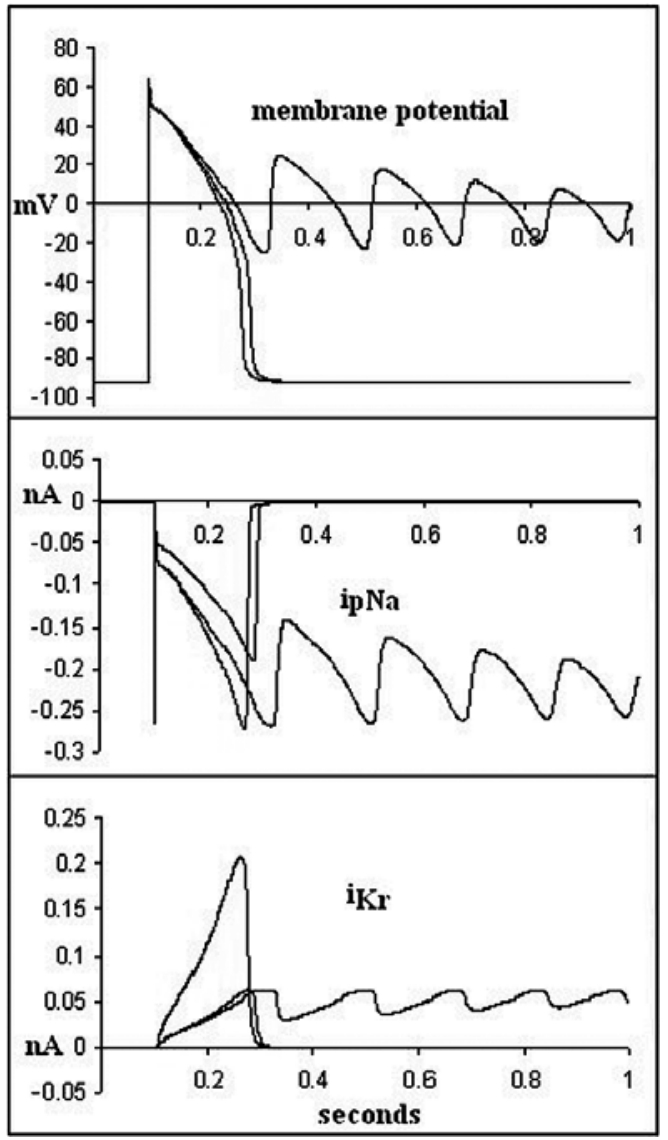

Fig. 5. Computations similar to those shown in Fig. 4, except that in this case inhibition of persistent sodium current is added to that of $i_{\mathrm{Kr}}$ to restore smooth repolarization. This result mimics the action of ranolazine (Ref. 26, but recomputed using COR).

sodium. When this produces sufficient outward $\mathrm{Na}^{+}-\mathrm{Ca}^{2+}$ exchange current (at about $280 \mathrm{~s}$ ) the model cell repolarizes and intracellular sodium falls again until repolarization failure recurs at about $440 \mathrm{~s}$. This cycle repeats itself indefinitely.

The exact mechanisms of arrhythmia during sodium overload conditions, such as those occurring during ischemia and heart failure, remain to be worked out. The mechanism reproduced here is just one example of a possible process by which arrhythmia could occur.

\section{Linking levels: building the virtual heart}

Although many of the mechanisms of cardiac arrhythmia can be studied and modelled at the cellular level, the question whether an arrhythmia is likely to be fatal requires analysis at multicellular levels, including that of the whole organ. A few ectopic beats, or a period of tachycardia may be survived, but if a re-entrant arrhythmia breaks down into fibrillation then it is usually fatal. The incorporation of the cellular models 


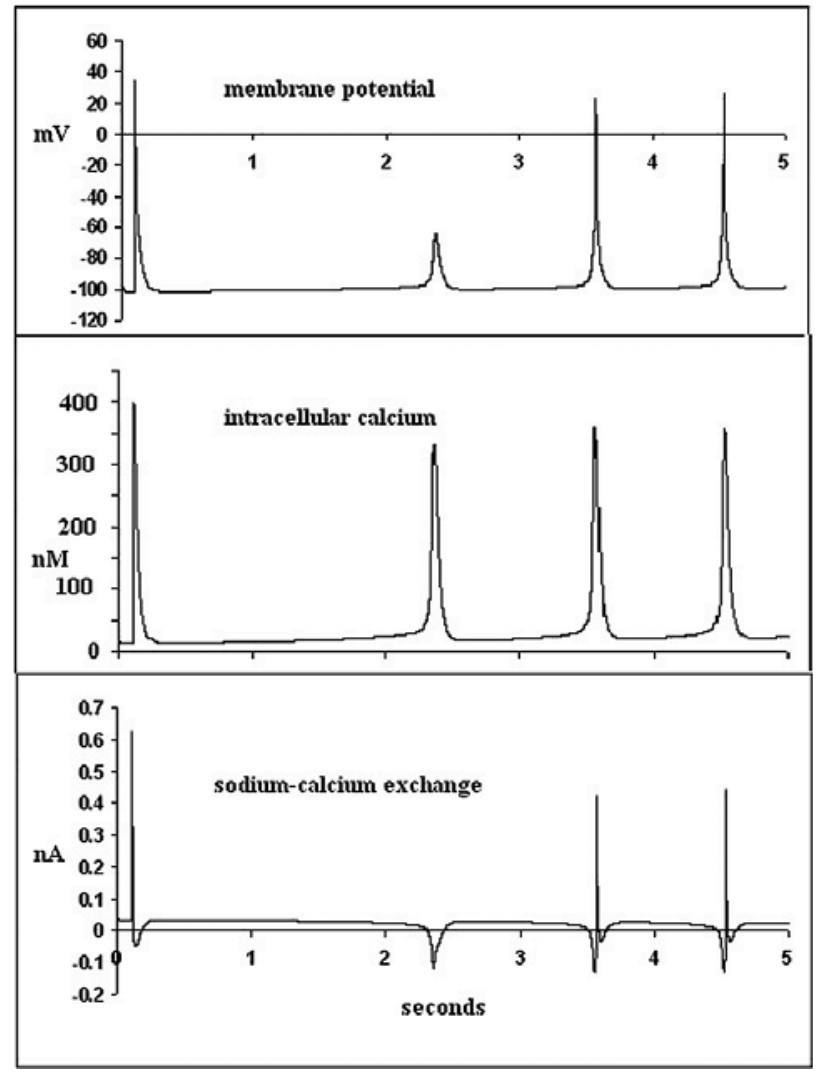

Fig. 6. The atrial cell model of Earm and Noble (28) has been subjected to sodium loading to raise intracellular sodium to a level $(14 \mathrm{mM})$ at which intracellular calcium oscillations occur. The first action potential (top trace) in this series was in response to an electrical stimulus. The subsequent action potentials are generated by calcium oscillations (middle trace). The inward current produced by sodium-calcium exchange (bottom trace) in response to each calcium transient is sufficient to reach threshold for action potential initiation, except for the first calcium release which generates a sub-threshold depolarization, DAD (Ref. 16, but recomputed using COR).

into models of cardiac tissue and of the whole organ is therefore essential. I have been privileged to collaborate with several of the key people involved in extending cardiac modelling to levels higher than the cell. The earliest work was with Raimond Winslow who used the Connection Machine at Minnesota, a huge parallel computer with 64,000 processors. We were able to construct models in which up to this number of cell models were connected together to form $2 \mathrm{D}$ or $3 \mathrm{D}$ blocks of atrial or ventricular tissue. This enabled us to study the factors determining whether ectopic beats generated during sodium overload would propagate across the tissue (32) and to study the possible interactions between sinus node and atrial cells (33).

Extension to the level of the whole organ came in collaboration with the University of Auckland where Peter Hunter, Bruce Smaill and their colleagues in bioengineering and physiology constructed the first
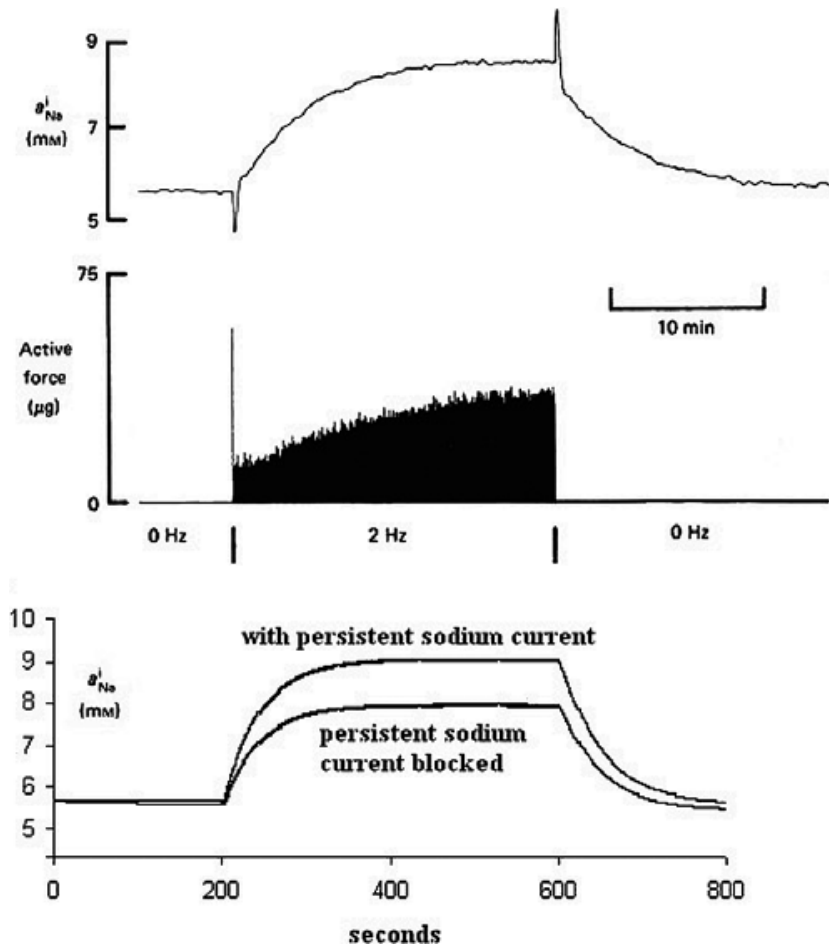

Fig. 7. Top: intracellular sodium activity and tension recorded in a cardiac Purkinje fibre during rest and during a period of repetitive stimulation at $2 \mathrm{~Hz}$ (28). Bottom: reconstruction using a model ventricular cell showing intracellular sodium concentration (25). The rise in intracellular sodium is similar to that seen experimentally. The same computation was then performed after $100 \%$ inhibition of persistent sodium current. The rise in sodium concentration is significantly reduced.

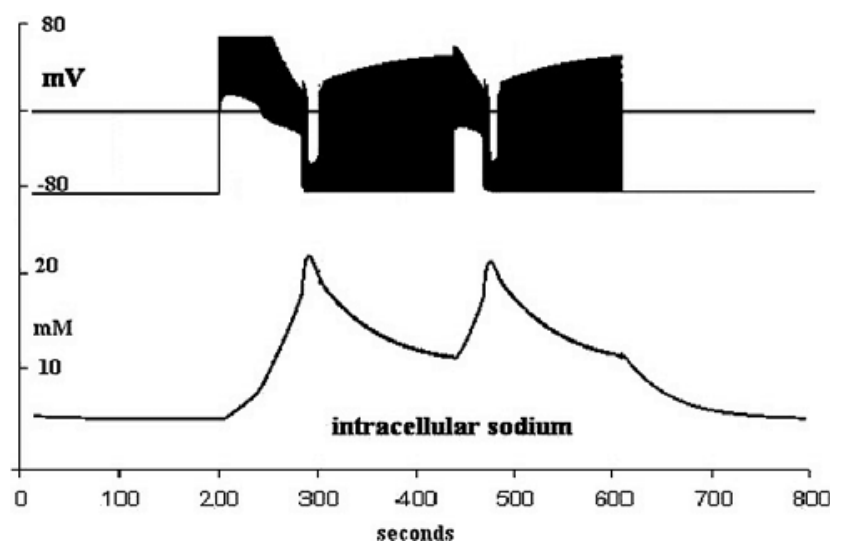

Fig. 8. Increasing the persistent sodium current in the model by $25 \%$ causes repolarization failure leading to a large rise in intracellular sodium. Intracellular sodium then oscillates slowly as a consequence of interactions between sodium and the membrane currents involved in repolarization. As yet, there is no experimental evidence for such slow oscillations during sodium overload. These computations therefore only demonstrate the possibility of such instability. 
anatomically-detailed models of a whole ventricle, including mechanics. These models include fibre orientations and sheet structure $(34,35)$, and have been used to incorporate the cellular models in an attempt to reconstruct the electrical and mechanical behavior of the whole organ.

This work includes simulation of the activation wave front $(36,37)$. This wave front is heavily influenced by cardiac ultra-structure, with preferential conduction along the fiber-sheet axes, and the results correspond well with that obtained from multi-electrode recording from dog hearts in situ. Accurate reconstruction of the depolarization wavefront promises to provide reconstruction of the early phases of the ECG to complement work already done on the late phases (15) and as the sinus node, atrium, and conducting system are incorporated into the whole heart model (38) we can look forward to the first example of reconstruction of a complete physiological process from the level of protein function right up to routine clinical observation.

The whole ventricular model has already been incorporated into a virtual torso (39), including the electrical conducting properties of the different tissues, to extend the external field computations to reconstruction of multiple-lead chest and limb recording. Incorporation of biophysically detailed cell models into whole organ models $(35,37,40,41)$ is still at an early stage of development, but it is essential to attempts to understand heart arrhythmias. So also is the extension of modelling to human cells $(42,43)$.

Work at the level of the whole ventricle has progressed rapidly as the necessary computing power has become available. This includes reconstructing some of the arrhythmic processes occurring during ischemia (44), the mechanisms of breakdown into fibrillation (45), modelling of the coronary circulation (46), and the mechanisms of defibrillation (41).

The multicellular and whole organ models are beginning also to be used in understanding the actions of drugs. A good example of this work within the BioSim network comes from Arun Holden's laboratory in Leeds. Figure 9 shows reconstruction of the spread of ectopic excitation in a model of the left ventricular wall. This work has been used to define the liminal volume necessary for an ectopic focus to initiate a fully conducted wave of excitation.

These models have been used to study the mechanisms of arrhythmogenesis $(47,48)$ and to study the actions of drugs such as $d$-sotalol on propagation (47). We can therefore look forward to testing the actions of drugs at multiple levels including that of the whole heart.

One of the main causes of cardiac arrhythmia is ischemia. Ischemia displays a high degree of hetero- geneity so that multicellular simulations are necessary to represent the full impact of ischemia. Electrophysiological properties vary not only with time postocclusion, but also spatially. Due to diffusion of ions and metabolites, the core of the tissue suffering from a lack of flow, that is the central ischemic zone (CIZ), is surrounded by border zones (BZ), which comprise progressive changes in electrophysiological properties between the healthy and ischemic regions. Experimental measurements of $\left[\mathrm{K}^{+}\right]_{0}$, oxygen, and metabolite distribution in the ischemic area $(49-53)$ were used by Ferrero et al. (54) to develop the 2D model of regional ischemia depicted in Fig. 10A, which included the first electrophysiologically detailed model of the BZ. Ischemia was represented by the effects of hyperkalemia, acidosis, and hypoxia on $\left[\mathrm{K}^{+}\right]_{\mathrm{o}}, \mathrm{I}_{\mathrm{Na}}, \mathrm{I}_{\mathrm{CaL}}$, and $\mathrm{I}_{\mathrm{K}(\mathrm{ATP})}$, at levels corresponding to 10 -min post-occlusion.

The severity of ischemic changes is most pronounced within the CIZ and decreases progressively in the BZ. The varying levels of $\left[\mathrm{K}^{+}\right]_{\mathrm{o}}, \mathrm{I}_{\mathrm{Na}}, \mathrm{I}_{\mathrm{CaL}}$ and $\mathrm{I}_{\mathrm{K}(\mathrm{ATP})}$ in the ischemic region result in a significant dispersion of refractoriness and of conduction velocity by the mechanisms explained in (44). Figure 10B shows the spatial variation of conduction velocity, APD, and effective refractory period in the border zone, as quantified by Ferrero et al. (54). Dispersion of refractoriness and of conduction velocity in regional ischemia provides the substrate for the establishment of reentrant circuits, the main mechanism of arrhythmogenesis following coronary occlusion $(53,55,56)$.

Figure 10C illustrates the initiation of a figure-ofeight reentry in this model of regional ischemia. Following pacing stimulation, a premature stimulus is applied at the bottom border of the 2D sheet. The premature stimulus elicits a wavefront, which propagates through the healthy region as well as the BZs, but blocks at the CIZ where refractoriness is extended. Meanwhile, tissue in the CIZ recovers, allowing reentry of the wavefront from the top, and the establishment of a figure-of-eight reentrant circuit, a pattern similar to the one observed experimentally $(57-60)$. Simulation results shows that the degree of activation of the $\mathrm{IK}_{\text {(ATP) }}$ plays an important role in vulnerability to reentry in regional ischemia $(54,61)$.

Simulation of disease states like ischemia will enable the study of drug interactions with various forms of disease-induced arrhythmia. One of the problems with computation at this level is that of computing resources. Even quite short simulations can require many hours of time on supercomputers. Work is progressing in attempting to solve these problems using more powerful computers and using networks of computers. We can therefore look forward to the day when it will be 

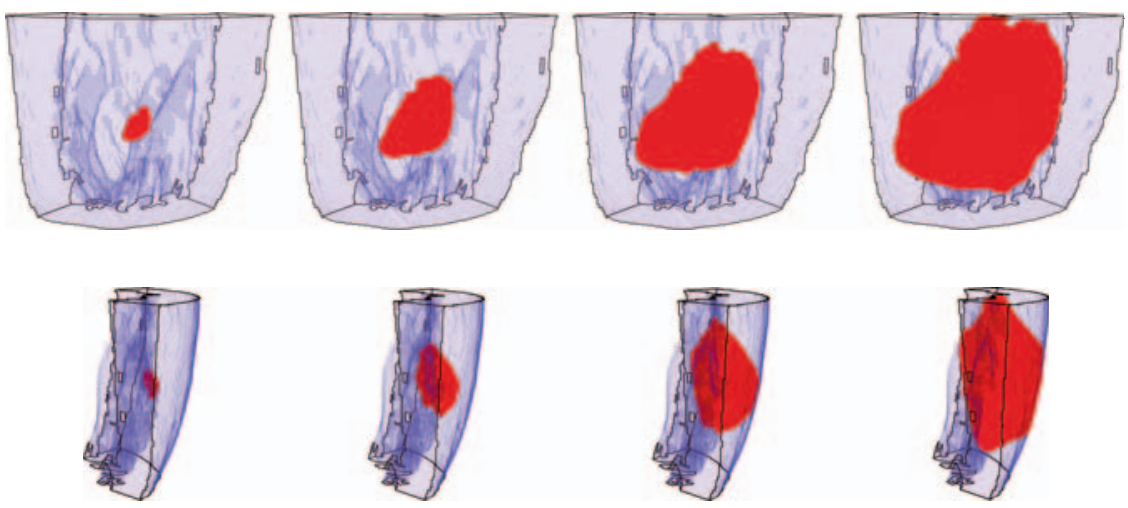

$$
t=10 \mathrm{~ms}
$$

$$
t=25 \mathrm{~ms}
$$

$$
t=40 \mathrm{~ms}
$$

$$
t=55 \mathrm{~ms}
$$

Fig. 9. Snapshots of orthotropic propagation of a single wave through a wedge model of the human end-diastolic (resting) left ventricular free wall, from an ectopic focus located on the endocardial surface. Spatially heterogeneous excitation kinetics are described using the ten Tusscher-Noble-Noble-Panfilov model (40), with endocardial, midmyocardial, and epicardial tissue occupying approximately equal fractions of the transmural distance. The diffusion coefficient in the fiber axis direction was set to

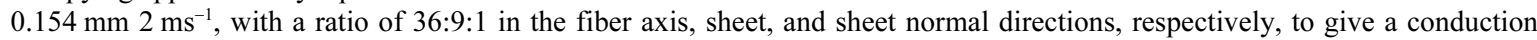
velocity ratio of $6: 3: 1$. Top panels show a view from the epicardial aspect, and bottom panels show a transmural view with the endocardium on the left and the epicardium on the right. The spatial extent of the wedge geometry is indicated in light blue, and excited tissue is in red. Times indicate duration since initial propagation from the ectopic focus. The architecture of the ventricular wall tissue results in complex wavefront geometries due to the rotational orthotropy inherent in the tissue. The wedge geometry and architecture was extracted from a human DT-MRI dataset provided by P.A. Helm and R.L. Winslow at the Center for Cardiovascular Bioinformatics and Modeling and E. McVeigh at the National Institute of Health (from Ref. 43 ).
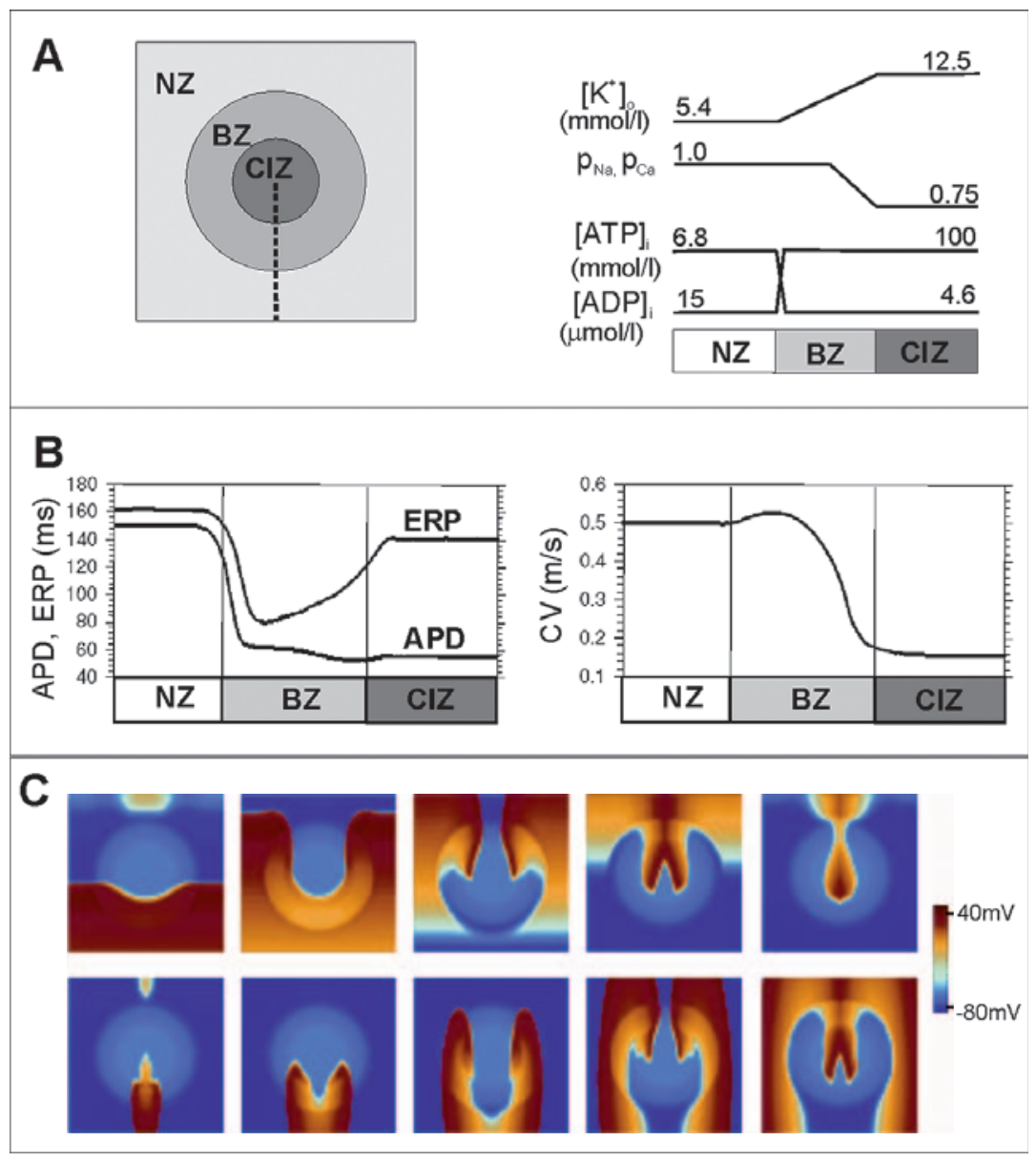

Fig. 10. Arrhythmogenesis in regional ischemia. Panel A: Schematic of the 2D model of regionally ischemic tissue (left) and the spatial variations in extracellular potassium concentration $\left(\left[\mathrm{K}^{+}\right]_{\mathrm{o}}\right)$, intracellular ATP and ADP concentrations $\left([\mathrm{ATP}]_{\mathrm{i}}\right.$ and $\left.[\mathrm{ADP}]_{\mathrm{i}}\right)$, and the degree of inhibition of the maximum conductances of the $\mathrm{Na}^{+}$and $\mathrm{Ca}^{2+}$ currents in the central ischemic zone (CIZ), border zone (BZ), and normal zone (NZ) (right). Panel B: Spatial variation in the effective refractory period and action potential duration (left) and in the longitudinal conduction velocity (right) along the dashed line depicted in panel A, left. Panel C: Snapshots of transmembrane potential distribution at different instants of time following the delivery of a premature stimulus at $\mathrm{CI}=210 \mathrm{~ms}$ in the lower part of the 2D sheet illustrated in panel A. Snapshots are separated by $50 \mathrm{~ms}$; the first corresponds to $50 \mathrm{~ms}$ after the delivery of the premature stimulus. Modified from Ferrero et al. (45). 
possible to have a complete suite of simulations in drug discovery and drug-testing running all the way from drug-receptor interactions to function in the whole organ.

\section{Acknowledgments}

Work in the author's laboratory is supported by EU FP6 BioSim network of excellence, EU FP7 PreDiCT project, The Wellcome Trust and the British Heart Foundation.

\section{References}

1 Noble D. Cardiac action and pacemaker potentials based on the Hodgkin-Huxley equations. Nature. 1960;188:495-497.

2 Noble D. A modification of the Hodgkin-Huxley equations applicable to Purkinje fibre action and pacemaker potentials. J Physiol. 1962;160:317-352.

3 Hutter OF, Noble D. Rectifying properties of heart muscle. Nature. 1960;188:495.

4 Hall AE, Hutter OF, Noble D. Current-voltage relations of Purkinje fibres in sodium-deficient solutions. J Physiol. 1963; 166:225-240.

5 Noble D. Electrical properties of cardiac muscle attributable to inward-going (anomalous) rectification. J Cell Comp Physiol. 1965;66 Suppl 2:127-136.

6 Hodgkin AL, Huxley AF. A quantitative description of membrane current and its application to conduction and excitation in nerve. J Physiol. 1952;117:500-544.

7 Weidmann S. Effect of current flow on the membrane potential of cardiac muscle. J Physiol. 1951;115:227-236.

8 Cole KS, Curtis HJ. Electric impedance of the squid giant axon during activity. J Gen Physiol. 1939;22:649-670.

9 McAllister RE, Noble D, Tsien RW. Reconstruction of the electrical activity of cardiac Purkinje fibres. J Physiol. 1975;251: $1-59$.

10 DiFrancesco D, Noble D. A model of cardiac electrical activity incorporating ionic pumps and concentration changes. Philos Trans R Soc Lond B Biol Sci. 1985;307:353-398.

11 Noble D. The music of life. Oxford: Oxford University Press; 2006.

12 Noble D, Colatsky TJ. A return to rational drug discovery: computer-based models of cells, organs and systems in drug target identification. Emerging Therapeutic Targets. 2000;4:3949.

13 Chen Q, Kirsch GE, Zhang D, Brugada R, Brugada J, Brugada P, et al. Genetic basis and molecular mechanism for idiopathic ventricular fibrillation. Nature. 1998;392:293-296.

14 Clancy CE, Rudy Y. Linking a genetic defect to its cellular phenotype in a cardiac arrhythmia. Nature. 1999;400:566-569.

15 Antzelevitch C, Nesterenko VV, Muzikant AL, Rice JJ, Chien $\mathrm{G}$, Colatsky T. Influence of transmural gradients on the electrophysiology and pharmacology of ventricular myocardium. Cellular basis for the Brugada and long-QT syndromes. Philos Trans R Soc Lond A. 2001;359:1201-1216.

16 Noble D, Varghese A, Kohl P, Noble PJ. Improved guinea-pig ventricular cell model incorporating a diadic space, $\mathrm{iKr}$ and $\mathrm{iKs}$, and length- and tension-dependent processes. Can J Cardiol. 1998;14:123-134.

17 Bril A, Faivre JF, Forest MC, Cheval B, Gout B, Linee P, et al. Electrophysiological effect of BRL-32872, a novel antiarrhythmic agent with potassium and calcium channel blocking properties, in guinea pig cardiac isolated preparations. J Pharmacol Exp Ther. 1995;273:1264-1272.

18 Watanabe Y, Kimura J. Inhibitory effect of amiodarone on $\mathrm{Na}+/ \mathrm{Ca} 2+$ exchange current in guinea-pig cardiac myocytes. $\mathrm{Br}$ J Pharmacol. 2000;131:80-84.

19 Kiyosue T, Arita M. Late sodium current and its contribution to action potential configuration in guinea pig ventricular myocytes. Circ Res. 1989;64:389-397.

20 Maltsev VA, Sabbah HN, Higgins RSD, Silverman N, Lesch M, Undrovinas AI. Novel, ultraslow inactivating sodium current in human ventricular myocytes. Circulation. 1998;98:2545-2552.

21 Sakmann BFAS, Spindler AJ, Bryant SM, Linz KW, Noble D. Distribution of a persistent sodium current across the ventricular wall in guinea pigs. Circ Res. 2000;87:910-914.

22 Maltsev VA, Undrovinas AI. A multi-modal composition of the late $\mathrm{Na}+$ current in human ventricular cardiomyocytes. Cardiovasc Res. 2006;69:116-127.

23 Belardinelli L, Shryock J, Fraser H. Inhibition of the late sodium current as a potential cardioprotective principle: effects of the selective late sodium current inhibitor, ranolazine. Heart. 2006; 92:iv6-iv10.

24 Bottino D, Penland RC, Stamps A, Traebert M, Dumotier B, Georgieva A, et al. Preclinical cardiac safety assessment of pharmaceutical compounds using an integrated systems-based computer model of the heart. Prog Biophys Mol Biol. 2006; 90:414-443.

25 Undrovinas AI, Belardinelli L, Nidas A, Undrovinas RN, Sabbah HN. Ranolazine improves abnormal repolarization and contraction in left ventricular myocytes of dogs with heart failure by inhibiting late sodium current. J Cardiovasc Electrophysiol. 2006;17:S169-S177.

26 Noble D, Noble PJ. Late sodium current in the pathophysiology of cardiovascular disease: consequences of sodium-calcium overload. Heart. 2006; 92:iv1-iv5.

27 Noble D, Varghese A. Modeling of sodium-calcium overload arrhythmias and their suppression. Can J Cardiol. 1998;14:97100.

28 Earm YE, Noble D. A model of the single atrial cell: relation between calcium current and calcium release. Proc R Soc Lond B Biol Sci. 1990;240:83-96.

29 Stern MD. Theory of excitation-contraction coupling in cardiac muscle. Biophys J. 1992;63:497-517.

30 Sher A, Hinch R, Noble PJ, Gavaghan D, Noble D. The role of $\mathrm{Na}^{+} / \mathrm{Ca}^{2+}$ exchangers in $\mathrm{Ca}^{2+}$ dynamics in ventricular myocytes. Prog Biophy Mol Biol. 2007;96:377-398.

31 Boyett MR, Hart G, Levi AJ, Roberts A. Effects of repetitive activity on developed force and intracellular sodium in isolated sheep and dog Purkinje fibres. J Physiol. 1987;388:295-322.

32 Winslow R, Varghese A, Noble D, Adlakha C, Hoythya A. Generation and propagation of ectopic beats induced by spatially localized Na-K pump inhibition in atrial network models. Proc Biol Sci. 1993;254:55-61.

33 Noble D, Brown HF, Winslow R. Propagation of pacemaker activity: interaction between pacemaker cells and atrial tissue. In: Huizinga JD, editor. Pacemaker activity and intercellular 
communication. CRC Press; 1995. p. 73-92.

34 Hooks DA, Tomlinson KA, Marsden SG, LeGrice IJ, Smaill BH, Pullan AJ, et al. Cardiac microstructure: implications for electrical propagation and defibrillation in the heart. Circ Res. 2002;91:331-338.

35 Crampin EJ, Halstead M, Hunter PJ, Nielsen P, Noble D, Smith $\mathrm{N}$, et al. Computational physiology and the physiome project. Exp Physiol. 2004;89:1-26.

36 Smith NP, Mulquiney PJ, Nash MP, Bradley CP, Nickerson DP, Hunter PJ. Mathematical modelling of the heart: cell to organ. Chaos, Solitons and Fractals. 2001;13:1613-1621.

37 Noble D. Modelling the heart: from genes to cells to the whole organ. Science. 2002;295:1678-1682.

38 Garny A, Kohl P, Noble D, Hunter PJ. 1D and 2D models of the origin and propagation of cardiac excitation from the sino-atrial node into the right atrium. Philos Trans R Soc Lond B Biol Sci. 2000;355, abstracts.

39 Bradley CP, Pullan AJ, Hunter PJ. Geometric modeling of the human torso using cubic Hermite elements. Ann Biomed Eng. 1997;25:96-111.

40 Noble D. The rise of computational biology. Nat Rev Mol Cell Biol. 2002;3:460-463.

41 Trayanova N, Eason J, Aguel F. Computer simulations of cardiac defibrillation: A look inside the heart. Computing and Visualization in Science. 2002;4:259-270.

42 Nygren A, Fiset C, Firek L, Clark JW, Lindblad DS, Clark RB, et al. A Mathematical model of an adult human atrial cell: the role of $\mathrm{K}^{+}$currents in repolarization. Circ Res. 1998;82:63-81.

43 Ten Tusscher KHWJ, Noble D, Noble PJ, Panfilov AV. A model of the human ventricular myocyte. Am J Physiol. 2004;286: H1573-H1589.

44 Rodriguez B, Trayanova N, Noble D. Modeling cardiac ischemia. Ann N Y Acad Sci. 2006;1080:395-414.

45 Panfilov A, Kerkhof P. Quantifying ventricular fibrillation: in silico research and clinical implications. IEEE Trans Biomed Eng. 2004;51:195-196.

46 Smith NP, Pullan AJ, Hunter PJ. An anatomically based model of transient coronary blood flow in the heart. SIAM Journal on Applied Mathematics. 2001;62:990-1018.

47 Benson AP. Computational electromechanics of the mammalian ventricles. In: Biological Sciences, vol. PhD. Leeds: University of Leeds; 2006.

48 Holden AV, Aslanidi OV, Benson AP, Clayton RH, Halley G, Li $\mathrm{P}$, et al. The virtual ventricular wall: a tool for exploring cardiac propagation and arrhythmogenesis. J Biol Phys. 2006;32:355368.
49 Janse MJ, Cinca J, Morena H, Fiolet JW, Kleber AG, de Vries GP, et al. The 'border zone' in myocardial ischemia. An electrophysiological, metabolic, and histochemical correlation in the pig heart. Circ Res. 1979;44:576-588.

50 Walfridson H, Odman S, Lund N. Myocardial oxygen pressure across the lateral border zone after acute coronary occlusion in pig heart. Adv Exp Med Biol. 1985;191:203-210.

51 Coronel R. Distribution of extracellular potassium during myocardial ischemia, vol. PhD. University of Amsterdam: PhD thesis; 1988.

52 Coronel R, Fiolet JW, Wilms-Schopman FJ, Schaapherder AF, Johnson TA, Gettes LS, et al. Distribution of extracellular potassium and its relation to electrophysiologic changes during acute myocardial ischemia in the isolated perfused porcine heart. Circulation. 1988;77:1125-1138.

53 Coronel R. Heterogeneity in extracellular potassium concentration during early myocardial ischaemia and reperfusion: implications for arrhythmogenesis. Cardiovasc Res. 1994;28:770-777.

54 Ferrero JMJR, Trenor B, Rodriguez B, Saiz J. Electrical activity and reentry during acute regional myocardial ischemia: insights from simulations. International Journal of Bifurcation and Chaos. 2003;13:1-13.

55 Pogwizd SM, Corr PB. Electrophysiologic mechanisms underlying arrhythmias due to reperfusion of ischemic myocardium. Circulation. 1987;76:402-426.

56 Wit AL, Janse MJ. The ventricular arrhythmias of ischemia and infarction: electrophysiological mechanisms. Mount Kisco: Futura Publishing Co; 1993.

57 Janse MJ, van Capelle FJ, Morsink H, Kleber AG, WilmsSchopman F, Cardinal R, et al. Flow of 'injury current' and patterns of excitation during early ventricular arrhythmias in acute regional myocardial ischemia in isolated porcine and canine hearts. Circ Res. 1980;47:151-165.

58 Janse MJ, Kleber AG. Electrophysiological changes and ventricular arrhythmias in the early phase of regional myocardial ischemia. Circ Res. 1981:49:1069-1081.

59 Kleber AG. Conduction of the impulse in the ischemia myocardium: implications for malignant ventricular arrhythmias. Experientia. 1987;43:1056-1061.

60 Costeas C, Peters NS, Waldecker B, Ciaccio EJ, Wit AL, Coromilas J. Mechanisms causing sustained ventricular tachycardia with multiple QRS morphologies. Circulation. 1997: 96:3721-3731.

61 Trenor B, Ferrero JMJR, Rodriguez B, Montilla F. Effect of pinacidil on reentrant arrhythmias generated during acute myocardial ischemia. Ann Biomed Eng. 2005;33:897-906. 